\title{
Journal of \\ Molecular Microbiology and Biotechnology
}

\section{Editor-in-Chief}

M.H. Saier, Jr., La Jolla, Calif.

\section{Senior Editors}

M.H. Brown, Adelaide, S.A.

J. Deutscher, Thiverval-Grignon

G. Gosset, Cuernavaca

P. Graumann, Marburg

M. Müller, Freiburg

D.H. Nies, Halle/Saale

R. Rabus, Oldenburg

F.X. Soberon, Cuernavaca

A. Vastermark, La Jolla, Calif.

\section{Editorial Board}

G. Bange, Marburg

F. Barras, Marseille

D.H. Bartlett, San Diego, Calif.

P. Beguin, Paris

I. Booth, Aberdeen

L.J. Brady, Gainesville, Fla.

Y.J. Chung, Jeonju

F.M. Commichau, Göttingen

P. Courvalin, Paris

C.J. Dorman, Dublin

J.S. Edwards, Albuquerque, N. Mex.

M.Y. Galperin, Bethesda, Md.

A. Goffeau, Louvain-la-Neuve

C.F. Gonzalez, Gainesville, Fla.

M. Gribskov, West Lafayette, Ind.

G.F. Hatfull, Pittsburgh, Pa.
P.J.F. Henderson, Leeds

S. Horinouchi, Tokyo

G. Klug, Giessen

G.L. Lorca, Gainesville, Fla.

J. Lutkenhaus, Kansas City, Kans.

W. Qin, Thunder Bay, Ont.

B.L. Reddy, Ontario, Calif.

V.S. Reddy, Boston, Mass.

K.C. Schuster, Lenzing

M.P. Shah, Ankleshwar

F. Titgemeyer, Münster

T.-T. Tseng, Kennesaw, Ga.

R. Vazquez-Duhalt, Cuernavaca

H. Wolf-Watz, Umea

$\mathrm{X}$. Zhou, Pullman, Wash. 
S. Karger

Medical and Scientific Publishers

Basel $\cdot$ Freiburg $\cdot$ Paris $\bullet$ London .

New York $\cdot$ Chennai $\cdot$ New Delhi $•$

Bangkok $\cdot$ Beijing $\cdot$ Shanghai $\cdot$ Tokyo $\cdot$

Kuala Lumpur $\cdot$ Singapore $\cdot$ Sydney

\section{Disclaimer}

The statements, opinions and data contained in this publication are solely those of the individual authors and contributors and not of the publisher and the editor(s). The appearance of advertisements in the journal is not a warranty, endorsement, or approval of the products or services advertised or of their effectiveness, quality or safety. The publisher and the editor(s) disclaim responsibility for any injury to persons or property resulting from any ideas, methods, instructions or products referred to in the content or advertisements.

Drug Dosage

The authors and the publisher have exerted every effort to ensure that drug selection and dosage set forth in this text are in accord with current recommendations and practice at the time of publication. However, in view of ongoing research, changes in government regulations, and the constant flow of information relating to drug therapy and drug reactions, the reader is urged to check the package insert for each drug for any change in indications and dosage and for added warnings and precautions. This is particularly important when the recommended agent is a new and/or infrequently employed drug.
All rights reserved

No part of this publication may be translated into other languages, reproduced or utilized in any form or by any means, electronic or mechanical, including photocopying, recording, microcopying, or by any information storage and retrieval system, without permission in writing from the publisher or in the case of photocopying, direct payment of a specified fee to the Copyright Clearance Center (see 'General Information')

(c) Copyright 2016 by S. Karger AG,

CH-4009 Basel (Switzerland)

Printed on acid-free and non-aging paper (ISO 9706)

\section{KARGER}


Journal of

Molecular Microbiology

and Biotechnology

No. 1-3

Synopsis

5 Anaerobic Microbial Degradation of Hydrocarbons: From Enzymatic Reactions to the Environment

Rabus, R. (Oldenburg); Boll, M. (Freiburg); Heider, J. (Marburg);

Meckenstock, R.U. (Neuherberg/Essen); Buckel, W. (Marburg); Einsle, O.

(Freiburg); Ermler, U. (Frankfurt); Golding, B.T. (Newcastle upon Tyne); Gunsalus, R.P. (Los Angeles, Calif.); Kroneck, P.M.H. (Konstanz); Krüger, M. (Hannover); Lueders, T. (Neuherberg); Martins, B.M. (Berlin); Musat, F.; Richnow, H.H. (Leipzig); Schink, B. (Konstanz); Seifert, J. (Stuttgart); Szaleniec, M. (Kraków); Treude, T. (Kiel/Los Angeles, Calif.); Ullmann, G.M. (Bayreuth); Vogt, C. (Leipzig); von Bergen, M. (Leipzig/Aalborg); Wilkes, H. (Oldenburg/Potsdam)

Review Articles

29 Structure and Function of Benzylsuccinate Synthase and Related Fumarate-Adding Glycyl Radical Enzymes Heider, J. (Marburg); Szaleniec, M. (Kraków); Martins, B.M. (Berlin); Seyhan, D.; Buckel, W. (Marburg); Golding, B.T. (Newcastle upon Tyne)

45 Ethylbenzene Dehydrogenase and Related Molybdenum Enzymes Involved in Oxygen-Independent Alkyl Chain Hydroxylation

Heider, J. (Marburg); Szaleniec, M. (Kraków); Sünwoldt, K. (Marburg); Boll, M. (Freiburg)

63 Anaerobic Degradation of $p$-Alkylated Benzoates and Toluenes

Rabus, R. (Oldenburg); Boll, M. (Freiburg); Golding, B.

(Newcastle upon Tyne); Wilkes, H. (Oldenburg/Potsdam)

76 Structure and Function of 4-Hydroxyphenylacetate Decarboxylase and Its Cognate Activating Enzyme

Selvaraj, B. (Berlin/Stockholm); Buckel, W. (Marburg); Golding, B.T. (Newcastle upon Tyne); Ullmann, G.M. (Bayreuth); Martins, B.M. (Berlin)

92 Anaerobic Degradation of Benzene and Polycyclic Aromatic Hydrocarbons

Meckenstock, R.U. (Essen); Boll, M. (Freiburg); Mouttaki, H. (Neuherberg); Koelschbach, J.S. (Essen/Neuherberg); Cunha Tarouco, P. (Neuherberg); Weyrauch, P.; Dong, X. (Essen/Neuherberg); Himmelberg, A.M. (Neuherberg)

119 Structure and Function of the Unusual Tungsten Enzymes Acetylene Hydratase and Class II Benzoyl-Coenzyme A Reductase

Boll, M.; Einsle, O. (Freiburg); Ermler, U. (Frankfurt); Kroneck, P.M.H. (Konstanz); Ullmann, G.M. (Bayreuth)

138 Metabolism of Hydrocarbons in $n$-Alkane-Utilizing Anaerobic Bacteria

Wilkes, H. (Oldenburg/Potsdam); Buckel, W. (Marburg); Golding, B.T.

(Newcastle upon Tyne); Rabus, R. (Oldenburg)

152 Activation of Acetone and Other Simple Ketones in Anaerobic Bacteria

Heider, J.; Schühle, K. (Marburg); Frey, J.; Schink, B. (Constance)
165 Fermentative Cyclohexane Carboxylate Formation in Syntrophus aciditrophicus

Boll, M.; Kung, J.W. (Freiburg); Ermler, U. (Frankfurt); Martins, B.M. (Berlin); Buckel, W. (Marburg)

180 Functional Gene Markers for Fumarate-Adding and Dearomatizing Key Enzymes in Anaerobic Aromatic Hydrocarbon Degradation in Terrestrial Environments von Netzer, F. (Neuherberg); Kuntze, K.; Vogt, C.; Richnow, H.H. (Leipzig); Boll, M. (Freiburg); Lueders, T. (Neuherberg)

195 Stable Isotope Probing Approaches to Study Anaerobic Hydrocarbon Degradation and Degraders

Vogt, C. (Leipzig); Lueders, T. (Neuherberg); Richnow, H.H. (Leipzig); Krüger, M. (Hannover); von Bergen, M. (Leipzig/Aalborg); Seifert, J. (Stuttgart)

211 Carbon and Hydrogen Stable Isotope Fractionation Associated with the Aerobic and Anaerobic Degradation of Saturated and Alkylated Aromatic Hydrocarbons Musat, F.; Vogt, C.; Richnow, H.H. (Leipzig)

227 Methanogenic Hydrocarbon Degradation: Evidence from Field and Laboratory Studies

Jiménez, N. (Hannover); Richnow, H.H.; Vogt, C. (Leipzig); Treude, T. (Los Angeles, Calif.); Krüger, M. (Hannover)

243 Author Index

244 Subject Index

No. 4

Research Articles

245 Clinical and Molecular Analysis of Klebsiella pneumoniae Causing Liver Abscess in China

Sun, Y. (Beijing/Linyi); Wu, H. (Haikou); Shen, D. (Beijing)

252 Mining of Ruminant Microbial Phytase (RPHY1) from Metagenomic Data of Mehsani Buffalo Breed: Identification, Gene Cloning, and Characterization

Mootapally, C.S. (Anand); Nathani, N.M. (Anand/Rajkot); Patel, A.K. (Ahmedabad); Jakhesara, S.J.; Joshi, C.G. (Anand)

261 Consistent Occurrence of Hydrocarbonoclastic Marinobacter Strains in Various Cultures of Picocyanobacteria from the Arabian Gulf: Promising Associations for Biodegradation of Marine Oil Pollution

Al-Wahaib, D.; Al-Bader, D.; Al-Shaikh Abdou, D.K.; Eliyas, M.; Radwan, S.S. (Safat)

269 Development of Chemically Defined Media to Express TrpAnalog-Labeled Proteins in a Lactococcus lactis Trp Auxotroph Shao, J. (Groningen); Marcondes, M.F.M.; Oliveira, V. (São Paulo); Broos, J. (Groningen)

\section{KARGER}

E-Mail karger@karger.com www.karger.com (c) 2016 S. Karger AG, Basel

Access to full text and tables of contents, including tentative ones for forthcoming issues: www.karger.com/mmb_issues 
277 Escherichia coli mazEF Toxin-Antitoxin System as a to Target Cell Ablation in Plants

Baldacci-Cresp, F.; Houbaert, A.; Metuor Dabire, A.; Mol, A.; Monteyne, D. El Jaziri, M.; Van Melderen, L.; Baucher, M. (Gosselies)

284 Differential CTX-M Expression from a Conserved Promoter: Role of Promoter-Associated Spacer Sequences Downstream of the bla $a_{\mathrm{CTX}-\mathrm{M}}$ Regulon

Liu, L. (Guiyang/Beijing); Zhang, X.; Yang, S. (Guiyang); Zhai, Y. (Beijing); Liu, W. (Guiyang); Wang, X. (Wuhan); Zhang, Z.; Gao, Z. (Beijing)

291 A Novel Phytase Derived from an Acidic Peat-Soil Microbiome Showing High Stability under Acidic Plus Pepsin Conditions Tan, H.; Wu, X.; Xie, L.; Huang, Z.; Peng, W.; Gan, B. (Chengdu)

\section{No. 5}

Research Articles

303 Expression and Biochemical Characterization of a Thermostable Branching Enzyme from Geobacillus thermoglucosidans

Ban, X.; Li, C.; Gu, Z.; Bao, C.; Qiu, Y.; Hong, Y.; Cheng, L.; Li, Z. (Wuxi)

312 Peptide Selectivity of the Proton-Coupled Oligopeptide Transporter from Neisseria meningitidis Sharma, N.; Aduri, N.G.; Iqbal, A.; Prabhala, B.K.; Mirza, O. (Copenhagen)

320 Transport and Catabolism of Carbohydrates by Neisseria meningitidis

Derkaoui, M. (Jouy-en-Josas/Paris); Antunes, A. (Paris); Nait Abdallah, J. (Jouy-en-Josas/Paris); Poncet, S.; Mazé, A.; Ma Pham, Q.M. (Jouy-en-Josas); Mokhtari, A. (Jouy-en-Josas/Guelma); Deghmane, A.-E. (Paris); Joyet, P. (Jouy-en-Josas); Taha, M.-K. (Paris); Deutscher, J. (Jouy-en-Josas/Paris)

333 The Number, Organization, and Size of Polymorphic Membrane Protein Coding Sequences as well as the Most Conserved Pmp Protein Differ within and across Chlamydia Species

Van Lent, S. (Ghent); Creasy, H.H. (Baltimore, Md.); Myers, G.S.A.

(Baltimore, Md./Sydney, N.S.W.); Vanrompay, D. (Ghent)

345 Systematic Exploration of the Glycoproteome of the Beneficial Gut Isolate Lactobacillus rhamnosus GG

Tytgat, H.L.P. (Leuven/Antwerp); Schoofs, G.; Vanderleyden, J. (Leuven);

Van Damme, E.J.M. (Ghent); Wattiez, R. (Mons); Lebeer, S.

(Leuven/Antwerp); Leroy, B. (Mons)

\section{No. 6}

Review

389 Systems Perspective of Morbillivirus Replication Kumar, N.; Barua, S.; Thachamvally, R.; Tripathi, B.N. (Hisar)

Research Articles

359 Soluble Prokaryotic Expression and Purification of Human Interferon Alpha-2b Using a Maltose-Binding Protein Tag Vu, T.T.T.; Jeong, B.; Krupa, M.; Kwon, U.; Song, J.-A.; Do, B.H.; Nguyen, M.T.; Seo, T.; Nguyen, A.N.; Joo, C.H.; Choe, H. (Seoul)

369 Transport and Catabolism of Pentitols by Listeria monocytogenes

Kentache, T.; Milohanic, E.; Cao, T.N. (Jouy-en-Josas); Mokhtari, A. (Jouy-en-Josas/Guelma); Aké, F.M.; Ma Pham, Q.M.; Joyet, P. (Jouy-en-Josas); Deutscher, J. (Jouy-en-Josas/Paris)

381 Analysis of 58 Families of Holins Using a Novel Program, PhyST

Kuppusamykrishnan, H.; Chau, L.M.; Moreno-Hagelsieb, G.; Saier Jr., M.H. (La Jolla, Calif.)

401 Proteomic Analyses Uncover the Mechanisms Underlying Antibiotic Resistance Differences among Three Acinetobacter baumannii Isolates

Wang, J.; Zhang, J.; Fu, Q.; Guo, S.; Ta, L.; Sun, P. (Hohhot)

414 Molecular Epidemiology of Human Metapneumovirus in Riyadh Province, Saudi Arabia H.M. Amer (Riyadh/Giza)

422 HA1-2-fljB Vaccine Induces Immune Responses against Pandemic Swine-Origin H1N1 Influenza Virus in Mice Kang, X.; Yang, Y.; Jiao, Y.; Song, H.; Song, L.; Xiong, D.; Wu, L.; Pan, Z.; Jiao, X. (Yangzhou)

Short Communication

410 Evidence of Interruption of the comM Gene in a Large Series of Clinical Isolates of Multidrug-Resistant Acinetobacter baumannii

Douraghi, M.; Jasemi, S.; Kodori, M.; Rahbar, M.; Boroumand, M.A. (Tehran) 